\title{
RILEM TC190-SBJ: development of recommendations on novel durability test methods for wet-applied curtain-wall sealants
}

\author{
Andreas T. Wolf
}

Published online: 23 September 2008

(C) RILEM 2008

\begin{abstract}
The paper discusses the work carried out over the past 7 years within RILEM Technical Committee 190-SBJ Service Life Prediction of Sealed Building and Construction Joints towards the development of RILEM Technical Recommendations (RTR) on durability test methods for wet-applied (gun-grade) curtain-wall sealants. This paper is published along with the two draft RTR methods to provide some insight into the discussions within the committee during the development and early evaluation of both methods. The two draft RTR methods define test and evaluation protocols for accelerated artificial weathering and natural outdoor weathering. Both draft RTR methods are based on a novel test specimen configuration that allows simultaneous exposure of the sealant to compression and extension during movement cycles. Because of the special test joint configuration, the sealant is exposed to variable extension and compression amplitudes along the joint axis, allowing an evaluation of the effect of different movement amplitudes on the sealant when taking weatherability considerations into account. The results of the initial evaluations indicate that the test methods are capable of differentiating between products with regard to their resistance to accelerated
\end{abstract}

\footnotetext{
A. T. Wolf $(\bowtie)$

Dow Corning GmbH, Rheingaustrasse 34, 65201 Wiesbaden, Germany

e-mail: andreas.wolf@dowcorning.com
}

or outdoor weathering and mechanical cycling. The type of failure and the changes in surface appearance observed during the test regimes are similar to those observed in actual service conditions. It is hoped that exposure studies based on these two new RTR methods will provide the underpinnings for the development of future ISO and ASTM durability test standards for building sealants.

Keywords Durability - Sealant .

Test method · Curtain-wall · RILEM TC190-SBJ

\section{Introduction}

Since the 1980s, the sealant industry has undergone rapid technological and structural changes. On the one hand, advancements in technology have enabled the launch of a multitude of new sealant products based on novel polymers, cure chemistries, and formulations. On the other hand, increasing competitive pressure and customers that are more demanding have required shorter product development cycles. Unlike the well-established sealants, which have been sold for more than 20 years based on the same formulation, the new sealant products do not have documented long-term performance histories. The sealants industry, therefore, urgently needs methods that can predict long-term performance rapidly and with assured reliability. Accelerated laboratory 
ageing experiments are the most promising method for acquiring durability information within the shortest possible time; however, a methodology for conducting and interpreting these experiments needs to be developed for prediction of long-term performance. Standardized exposure and evaluation protocols are also needed to benchmark the results obtained in accelerated artificial weathering against those observed in natural outdoor weathering exposures [1].

A first step towards this goal was achieved with the work of RILEM Technical Committee 139-DBS Durability of Building Sealants that culminated in the publication of an RTR method designed to evaluate the durability of curtain-wall sealants exposed to artificial weathering and mechanical cycling [2]. The validity of this test method was demonstrated by two separate studies, which showed that the method was able to differentiate between sealant products with regard to their resistance to accelerated weathering and mechanical cycling. Furthermore, the type of failure and the changes in surface appearance observed in both studies (using somewhat different test regimes, but otherwise standardized protocols) were similar to those observed on sealants exposed to actual service conditions [3, 4].

Discussions within RILEM TC190-SBJ, which was chartered to continue the work of TC139-DBS with regard to the development and verification of durability test methods for wet-applied (gun-grade) sealant products, showed that the test method proposed by TC139-DBS was lacking in some aspects. First, a test protocol was highly desirable that allowed simultaneous exposure to weathering and movement cycles. The TC139-DBS protocol, however, only allowed sequential exposure to weathering and movement cycles. Recent research, initiated within the RILEM TC139-DBS committee and continued under the auspices of TC190-SBJ committee, into the effects of accelerated artificial weathering combined with simultaneous or sequential strain cycling, indicated that degradation occurs more rapidly, when cyclic strain acts simultaneously with weathering [5]. Second, sealants tested according to the TC139-DBS protocol are undergoing cyclic mechanical strain based on the movement capability quoted by their manufacturer, which typically does not take long-term durability considerations into account. Rather, for wet-applied sealants, movement capability is typically rated based on either ISO 11600 [6] or ASTM C920 [7] classification schemes, which do not comprise any long-term durability assessment. Consequently, sealants often fail prematurely in the TC139-DBS RTR test method when exposed to cyclic movement at their rated movement capability, while they would have lasted substantially longer at a lower movement amplitude exposure. On the other hand, it is cumbersome attempting to use the TC139-DBS test protocol to determine the movement capability of sealants under accelerated weathering conditions, since this requires a whole series of specimens being exposed to different cyclic movement amplitudes. What is needed, thus, is a test method that allows an estimation of the movement capability of sealants while taking the effects of weathering and mechanical cycling directly into account.

In 2001, RILEM TC190-SBJ became aware of work that had been initiated by the Architectural Institute of Japan in November 2000 with the aim of developing an accelerated weathering test method for weatherproofing sealants. The Japanese TC members and experts presented the concept of their proposed test method along with the suggested experimental evaluation protocols and some preliminary conceptevaluation results at the TC190-SBJ meeting in Lyon, France [8]. At the same meeting, the French and German committee members and experts jointly made an alternative test method proposal, however, only the Japanese proposal addressed the two shortcomings of the TC139-DBS RTR method described above. Nevertheless, for the time being, all researchers involved agreed to pursue the initial evaluation of both methods under the auspices of TC190-SBJ.

Based on early results obtained with these two proposed methods (and some modifications of these methods) it became obvious within the next 2 years that the Japanese proposal provided a better balance of weathering and cyclic movement exposure and had the added benefit of overcoming some of the limitations of the previous TC139-DBS RTR test method [9]. Therefore, this review paper focuses only on the discussions and developments around the Japanese proposal, which ultimately led to the publication of the two draft TC190-SBJ RTR methods in this journal.

During the development of the RTR methods, TC190SBJ closely cooperated with the ISO TC59/SC8 
committee on Building Construction-Jointing Products. By holding meetings of TC190-SBJ in conjunction with plenary meetings or work group meetings of ISO TC59/SC8, technical discussions in both committees were able to cross-fertilize each other. Recently, ISO TC59/SC8 Work Group 6 (Durability Test Method) agreed to consider the TC190-SBJ draft RTR method on accelerated weathering as a basis for the development of a future International Standard [10]. Similarly, ASTM C24 Committee on Seals and Sealants had considered the TC190-DBS RTR method in the past as a basis for the development of the ASTM C1519 standard [11]. Therefore, it is hoped that the pre-normative work conducted under the auspices of RILEM TC190-SBJ (and, hopefully, its successor committee) will continue to provide the scientific underpinnings for the future development of both ISO and ASTM durability standards.

\section{Key considerations in the development of durability test methods for sealants}

During their entire service life, joint seals are exposed to cyclic mechanical strain and environmental degradation factors. Cyclic joint movement, sunlight, temperature variations (heat, cold) and moisture (water) are considered to be the primary environmental and service degradation factors leading to sealed joint failure. Weatherproofing joint seals in building façades are exposed to frequent cyclic movements. This joint movement imposes cyclic mechanical strain on the seal, which, depending on the exposure conditions and the construction design, can vary substantially in rate and amplitude.

Work on the durability test methods started with the premise that the accelerated artificial weathering regime should incorporate only the following four key ageing factors: solar radiation, moisture, temperature, and joint movement. With this limitation, the RILEM 190-SBJ committee realised the impossibility of incorporating all possible combinations of weathering and service factors into a single laboratorybased testing regime. The exposure of sealant specimens to artificial weathering and cyclic movement was intended to simulate the effect of a natural weathering environment on installed weatherproofing sealants exposed to outdoor weather and joint movement. The artificial weathering exposures were not meant to simulate the deterioration caused by localised environmental conditions, such as atmospheric pollution, biological attack or salt-water exposure.

Laboratory-based artificial weathering methods provide only approximations of natural outdoor weathering conditions. The results of artificial weathering tests depend on the choice of the experimental test parameters, such as light source, exposure temperatures, movement amplitudes, et cetera. In order to establish a reasonable assessment of the durability of sealants observed in artificial weathering tests as benchmarked against the durability expected in outdoor weathering, the committee decided to strongly recommend that control materials with known outdoor exposure durability should be included with each artificial weathering test.

Contrary to accelerated artificial weathering, for which only a simplified set of ageing factors can be considered, in outdoor exposures all weathering factors specific to the exposure site act in synergy. It is important to note that the site-specific weathering and degradation factors often include factors not considered in the artificial accelerated weathering regimes. Obviously, this issue limits the correlation of results obtained by artificial and outdoor weathering. The committee further acknowledged that the durability of sealants, as assessed by outdoor exposure, varies with geographic location of the site, exposure conditions, site-specific weathering and degradation factors, as well as seasonal and annual climate changes. In order to overcome this variability issue, the stability of materials in outdoor weathering is generally determined relative to controls with known performance exposed simultaneously, unless the stabilities of a series of materials exposed at the same time are being compared. Therefore, the committee decided to strongly recommend that control materials (sealants) with known durability should be included with each exposure test. Preferably, two control materials of similar composition and construction to the test specimens are to be used, one with relatively good durability and one with relatively poor durability.

The committee decided not to restrict the outdoor exposure to specific climates, but to allow exposures in any type of climate. However, when conducting outdoor weathering exposures, it is important to 
consider how well the test conditions reproduce property changes and failure modes associated with the end-use environments for the sealants to be tested. For example, if the use environment for the sealant being evaluated will include freeze/thaw cycling, specimen exposure in a northern climate may be advisable. Other use climates may require exposure of the specimens in locations where they are subjected to salt particulates (seashore) or industrial pollutants.

Joint movement is the preponderant service factor in the ageing of sealants, since it has a damaging effect both while the sealant is curing and after completion of the cure. Depending on the type of joints, sealants are exposed to various types and degrees of movement. Joint movements are induced by various factors, such as:

- Thermal expansion/contraction of construction components

- Shrinkage of construction components

- Settling of buildings

- Wind loads

- Service loads.

Some of these factors may occur simultaneously, exposing the sealed joint to a rather complex, stochastic, often three-dimensional movement pattern of overlapping shear and tensile movements. Such complex movement patterns are difficult to simulate and the analysis of its effect on the sealant properties is an even more challenging task. Therefore, for the purpose of developing the durability test standards, the RILEM 190-SBJ committee decided to consider only cyclic movement resulting primarily in tensile loads. This type of movement occurs most frequently in curtain-wall joints. However, even in this application, other movements, such as in shear, often occur simultaneously. A further narrowing of the scope of the initial test standard occurred when the committee decided to consider only elastic sealants, as defined by the ISO 11600 classification scheme.

\section{Novel test specimen design}

As mentioned before, the new specimen configuration proposed by the Japanese members and experts deviates completely from the design used in the TC139-DBS RTR or in any ISO TC59/SC8 standard in as far as the support elements (substrates) to which the sealant adheres are riveted to the base plate with pivoted hinges (see Fig. 1).

This configuration allows independent movement of the support elements. Once the sealant has been applied and cured in the joint formed by the support elements, insertion of a separator on the end of the joint will cause simultaneous extension and compression in different areas of the joint (see Fig. 2). As the elastic sealant acts like a spring, both support elements will experience the same degree of angular movement due to the insertion of the separator, provided the friction in the riveted hinges is negligible.

By extending one end of the joint test specimen, the other end gets compressed; thus, the test specimen can be exposed simultaneously to various degrees of extension and compression in a single movement. The inspection of the test specimen after a certain exposure time then allows for the evaluation of the type of damage (adhesive or cohesive failure as well as changes in surface appearance), the maximum depth of cracks, the crack density, and the location of the cracks (bulk or peripheral region) for a specific extension/compression value achieved along the length of the specimen. For instance, changes in specimen surface appearance may be reported for $0 \%, \pm 10 \%, \pm 20 \%$, and $\pm 30 \%$ tensile compression/ extension movements.

Note that the centre-section of the specimen, while not being exposed to tensile compression/extension movements, is exposed to a certain, but undefined,

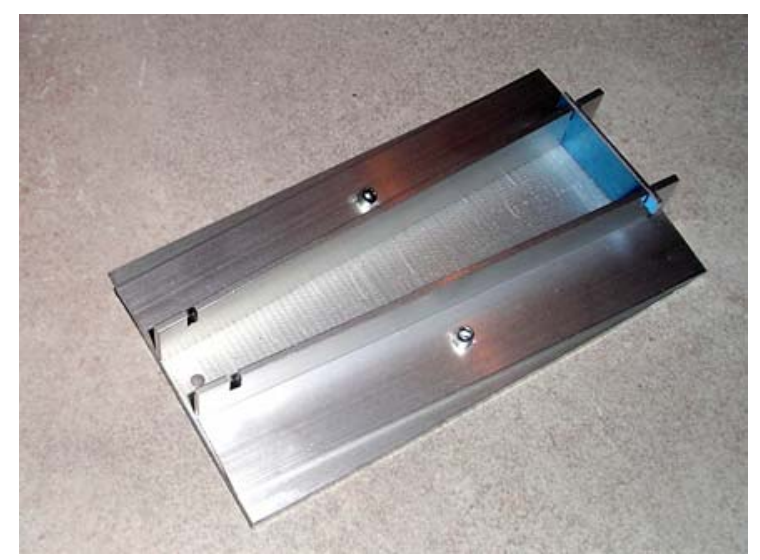

Fig. 1 Photo of support configuration (support elements riveted to support base plate) used for cyclic mechanical movement of sealant 


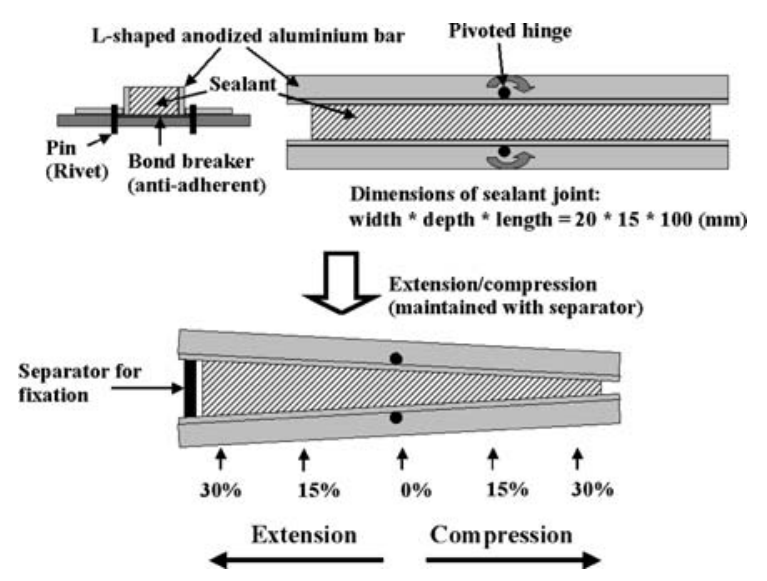

Fig. 2 Schematic drawing of test specimen: sealant in anodised aluminium support used for cyclic mechanical movement of sealant

amount of shear movement resulting from the displacement of sealant material in the compressed section of the specimen. Therefore, acceleration factors for mechanical movement cannot be derived from this test method due to the specimen design without the use of separate (static) controls. Figure 3 shows, as an example, the changes in surface appearance occurring on a test specimen. The dependency of the degree of surface degradation on the amplitude of the movement is readily visible. Note that the centre-section of the sealant is not completely free of cracks and that the direction of the cracks located in the centre-section indicates exposure to shear movements.

The novel specimen design can be used for both accelerated artificial and natural outdoor weathering

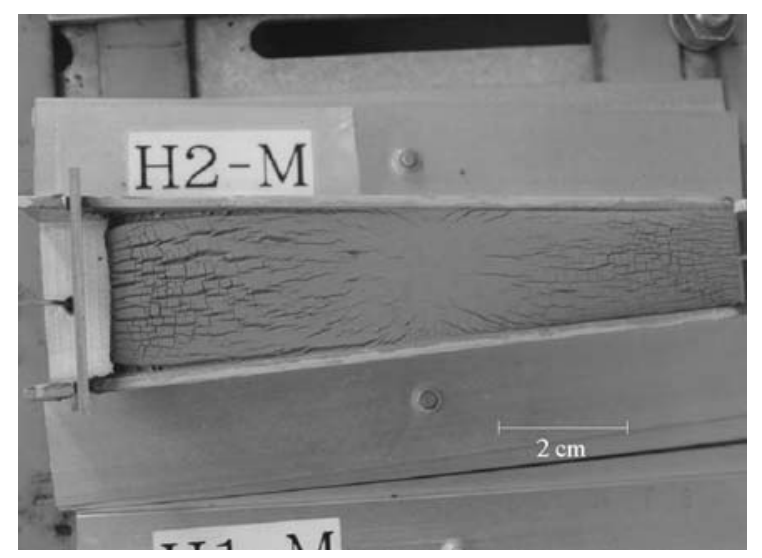

Fig. 3 Photo of weathered test specimen exposures. Within a single test specimen, sealant durability can be assessed for a range of movement amplitudes. The novel design requires less handling of the test specimen in order to achieve a certain movement exposure, since it allows simultaneous extension and compression of the sealant in different areas of a single test specimen. Because of the simplified handling of the specimen, the novel design enables simultaneous exposure of the test specimen to weathering and cyclic movement; however, it may also be employed in test protocols where these ageing factors act sequentially.

Recent research has shown that the type of backing material used in actual service joints has a considerable effect on sealed joint performance [12]. Studies are currently underway to investigate modifications of the test specimen design (increased joint depth) that allow evaluation of different backing materials as part of the durability assessment of integral sealed joint designs. Plans for further research are being discussed that will evaluate the applicability of the novel test specimen to studies of the effect of early joint movement (during sealant cure) on the durability of the sealed joint.

\section{Durability test concepts}

The basic durability test concepts were built on a proposal made in 1993 within the RILEM TC139DBS committee, as shown in Fig. 4. As previously within RILEM TC139-DBS, much discussion revolved around the question of how the sealants should be conditioned prior to exposing them to the durability cycles. Finally, the proposal to use the same conditioning methods as defined in ISO 11600 was widely supported, based on the assumption that this approach allowed direct comparison of the result of the durability cycles with the 'initial' properties as determined by the other test methods referenced in ISO 11600. For the time being, the committee decided to focus on static cure of the specimen (without movement) in a laboratory-controlled climate. Conditioning method A, i.e. 28 days at $(23 \pm 2)^{\circ} \mathrm{C}$ and $(50 \pm 5) \%$ relative humidity, as defined in various ISO standards on sealants, was again chosen as the default. As an alternative option for slower curing sealants, conditioning method B was also allowed. This method exposes the specimen 


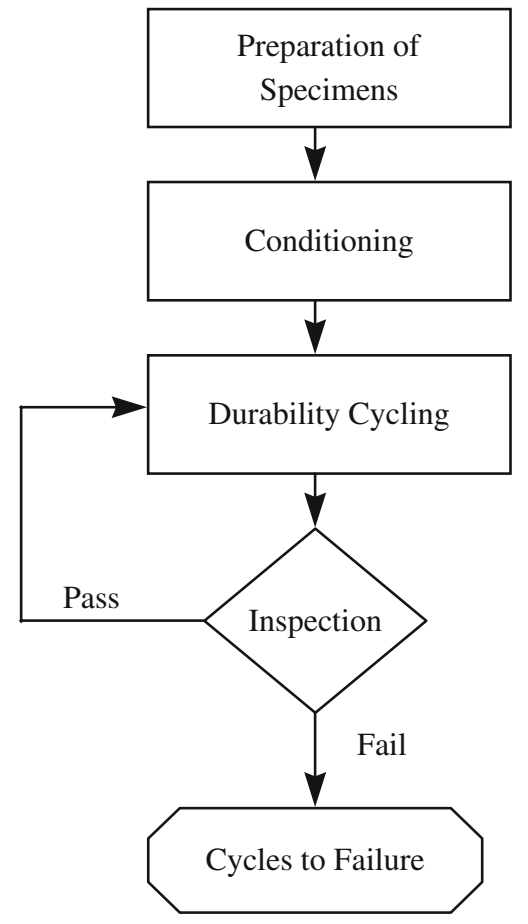

Fig. 4 Basic durability test concept

after completion of conditioning method $\mathrm{A}$ to an additional 21 days of alternating storage in dry heat and water immersion. Conditioning B is considered as a normal conditioning method using the influence of water and heat to accelerate the cure of the sealant. It is not part of the durability assessment of the sealant.

During the discussion of the durability exposure cycles, the committee decided to allow both, simultaneous and sequential, exposure options for weathering and enforced mechanical movement in the artificial weathering exposure. For the natural outdoor weathering exposure, the simplest protocol was one where weathering and mechanical cycling occurred simultaneously. Mechanical cycling was to be induced in the novel test specimen by extending or compressing one end of the test specimen with a suitable device, for instance a fully-automated cyclic movement machine, a tensile test machine, or a handoperated vice, and inserting a spacer (referred to in the RTR as 'separator' in order to differentiate this tool from the 'spacer' used during specimen preparation).

For the artificial weathering exposure, the following alternative exposure protocol options were defined: (a) Sequential exposure: Weathering is carried out for 6 weeks (default value) in an artificial weathering machine. Then the specimens are exposed to 6 weeks (default value) of movement cycles.

(b) Simultaneous exposure: The specimens are exposed to 6 weeks of movement and artificial weathering carried out simultaneously.

The combination of artificial weathering and mechanical movement regimes, carried out either sequentially or simultaneously, was considered to constitute an exposure cycle. After completion of each exposure cycle, the test specimens were to be visually examined for changes in appearance, cohesion and adhesion. The concept then was to repeat the degradation cycle as often as required to achieve a certain degradation. For outdoor weathering, inspections of the test specimens after completion of each durability cycle were to be replaced by inspections at regular 3-month intervals.

As with the RTR durability test method recommended by the 139-DBS committee in 2001, TC190SBJ decided to allow alternative options for the artificial light source and the method of water exposure in the accelerated weathering regime. The justification for this decision was primarily that of a largely inhomogeneous global installed equipment base. While in North America fluorescent ultraviolet sources had been commonly used, ${ }^{1}$ xenon-arc light sources see widespread use in Europe, and openflame carbon arc sources are still favoured in Asia, especially in Japan. Sealant durability assessment studies typically utilize automated artificial weathering equipment; however, there are substantial differences in the methods employed in these devices to achieve water or humidity exposure. The reasoning used by the committee for allowing certain options, while not including others, and for selecting the default methods is discussed below.

The degree of approximation to the spectral power distribution of the solar global radiation at the surface

\footnotetext{
${ }^{1}$ Currently, xenon arc light sources are used in North America to an equal extent or even more widely than fluorescent ultraviolet sources for laboratory accelerated weathering of sealants. The relevant ASTM standard practices and test methods no longer specify fluorescent ultraviolet sources without also specifying xenon arc sources and many laboratories prefer the xenon arc source.
} 
of the earth depends on the type of artificial light source [13]. Xenon-arc lamps with suitable filters are regarded as providing the best representation of the solar spectral irradiance at ground level and, therefore, are considered the default for the purpose of the RTR recommended by TC190-SBJ (for a discussion of the various artificial light sources and their relevance to sealant durability testing see [14-16]).

In some accelerated weathering devices, liquid water exposure is induced by condensation of humidity during a cooling phase. However, it has been found that for adequate heat transfer to occur during the condensation period, a specimen thickness of less than $20 \mathrm{~mm}$ is required (dimensions of the support included). ${ }^{2}$ Therefore, since the condensation process provided in accelerated weathering devices is generally not applicable to the type of sealant specimens tested, the recommended default in the RTR is to carry out wetting by water spray on the exposed specimen surface. However, the front surface water spray accessory in the fluorescent UV weathering device was not designed for this purpose and requires an unreasonable amount of pure (deionised) water for the wet period specified. Therefore, in order to comply with the default specification in the RTR, the equipment requires modification in order to allow recirculation of the water during the exposure period.

Immersion of the specimen in water is allowed as an optional alternative method of water exposure in the RTR. This decision by the committee recognizes the fact that a substantial portion of the installed base of automatic xenon-arc weathering devices utilizes water immersion instead of water spray. Round robin tests were carried out on a set of sealants within ISO TC59/SC8 in the past to address the comparability of results obtained with both methods. ${ }^{3}$ Data generated with these two methods of water exposure (spray or immersion) as part of the revision of ISO 11431 [17] showed acceptable correlation, although contributions to the various degradation mechanisms acting in the specimens (e.g. hydrolysis, thermal shock, leaching of formulation components, et cetera) can differ between these exposures. The degree of correlation

\footnotetext{
$\overline{2}$ See comments included in the footnotes of Ref. [2].

3 Round-robin tests were carried out in ISO TC59/SC8/WG05 during the time period 1996-1999.
}

between these two methods thus may vary depending on the specific sealant tested.

With the exception of the forced mechanical movement, the weathering and degradation factors acting on the sealant in outdoor exposure tests cannot be controlled. Consequently, the durability of sealants in actual outdoor use can differ substantially depending on the location, because of differences in solar radiation, moisture, temperature, pollutants, and other factors [18]. Discussions within the committee, therefore, focused primarily on what information needs to be recorded in the test report. Tests conducted in accordance with this RTR are used to evaluate the relative stability of sealants when exposed outdoors with mechanical movement. The committee recommended that the stability of the test sealants should be determined relative to controls with known performance (exposed simultaneously), unless the stabilities of a series of materials exposed at the same time are being compared.

Sealant colour may also affect durability, both in artificial and in natural weathering. Many pigments are very effective in absorbing solar radiation. Pigments may have either a stabilizing or destabilizing effect on the sealant with regard to its weatherability. Furthermore, photo-stabilizers and antioxidants may adsorb on certain pigments and, by doing so, be rendered ineffective [19]. Pigmented versions of the same sealant formulation may therefore show different resistance to artificial and natural weathering, as has been recently demonstrated for two differently coloured polysulfide sealants exposed to outdoor weathering [20]. Therefore, the colour of the sealant tested must be mentioned in the test report.

As discussed before, the 190-SBJ committee decided on test protocols in which exposure to mechanical cycling is carried out simultaneously with outdoor weathering and either simultaneously or sequentially with accelerated artificial weathering. Simultaneous extension and compression of different areas of the test specimen is achieved by extending or compressing one end of the test specimen with a suitable device, for instance a fully-automated cyclic movement machine, a tensile test machine, or a handoperated vice, and inserting a separator (spacer) upon completion of the operation in the extended end of the specimen. Various devices were deemed suitable for extending or compressing one end of the 
specimen and the committee decided intentionally not to restrict the nature of the device used for this task.

Finally, the committee discussed previous experimental findings that suggest that the results of laboratory exposure (artificial weathering) cannot be directly extrapolated to estimate an absolute rate of deterioration caused by natural weathering because the acceleration factor is material dependent and can be substantially different for each sealant material (polymer base) and for different formulations of the same material [21].

\section{Summary of the RTR durability test methods}

The two RILEM recommendations specify laboratory and outdoor exposure procedures for determining the effects of cyclic movement and artificial or natural weathering on cured, elastic weatherproofing joint sealants (one- or multi-component).

In these test procedures, test specimens are prepared in which the sealant to be tested adheres to two parallel support surfaces (substrates). The specimens are conditioned statically (no movement during cure) in a laboratory-controlled climate. The conditioned specimens are then exposed to artificial or natural weathering and cyclic movement.

In the artificial weathering procedure, weathering is carried out for 6 weeks (default value) in an artificial weathering machine. Then the specimens are exposed to 6 weeks (default value) of movement cycles. Alternatively, the specimens may be exposed to 6 weeks of movement and artificial weathering carried out simultaneously. The combination of artificial weathering and mechanical movement regimes, carried out either sequentially or simultaneously, constitutes an exposure cycle. After completion of each exposure cycle, the specimens are visually examined for changes in appearance, cohesion and adhesion. In the natural weathering protocol, the specimens are weathered by exposing them on a rack outdoors. At regular intervals (default value: every 3 months), the specimens are visually inspected for changes in appearance, cohesion and adhesion.

By extending or compressing one end of the test specimen with a suitable device and inserting a separator upon completion of the operation in the extended end of the specimen, forced mechanical cycling is induced in the specimen.

The visual inspection of the specimens after completion of each durability cycle or at regular intervals in the outdoor weathering protocol is done in their extended/compressed state (extension/compression up to the full rated movement capability of the sealant tested). The depth of any cohesive or adhesive flaw is determined according to the rules provided in ISO 11600 and the general condition of the sealant is reported. Schematic representations of the test procedures are shown in Figs. 5 and 6.

Default test parameters and, for some procedures, alternative options are defined in the technical recommendations. In cases of dispute, the default method is the reference method. The experimenter may deviate from the default values for the following test parameters, however, deviations from the default values must be highlighted in the test report:

(a) Support substrate-default: anodised aluminium

(b) L-shaped support element dimensions-default: $120 \mathrm{~mm} \times 20 \mathrm{~mm} \times 15 \mathrm{~mm} \times 2.0 \mathrm{~mm}$ (length $\times$ width $\times$ height $\times$ thickness)

(c) Sealed joint dimensions-default: $100 \mathrm{~mm} \times$ $20 \mathrm{~mm} \times 15 \mathrm{~mm}$ (length $\times$ width $\times$ depth)

(d) Conditioning method (A or B)—default: A

(e) Accelerated artificial weathering: Light source (xenon-arc, fluorescent UVA-340 lamp, openflame carbon arc)—default: xenon-arc; Weathering procedure (default period: 6 weeks): duration of artificial weathering, type of water exposure (spraying or immersion), the temperature of light exposure, the temperature of water exposure, the timing of light and moisture/water cycle-default values are specified for xenon arc/water-spray, xenon-arc/water immersion, fluorescent UVA-340/water-spray weathering and open-flame carbon arc/water spray weathering

(f) Natural outdoor weathering: Outdoor exposure-default: exposure rack with $45^{\circ}$ inclination angle facing the equator

(g) Mechanical cycling (default period: 6 weeks): amplitude and duration (number of cycles) default values are specified in the test procedure.

Finally, the committee decided to highlight the fact that the use of these RTR durability test methods 
Fig. 5 Schematic flowchart of artificial and natural weathering test procedures with simultaneous weathering and mechanical cycling

Fig. 6 Schematic flowchart of artificial weathering test procedure with sequential periods of weathering and mechanical cycling
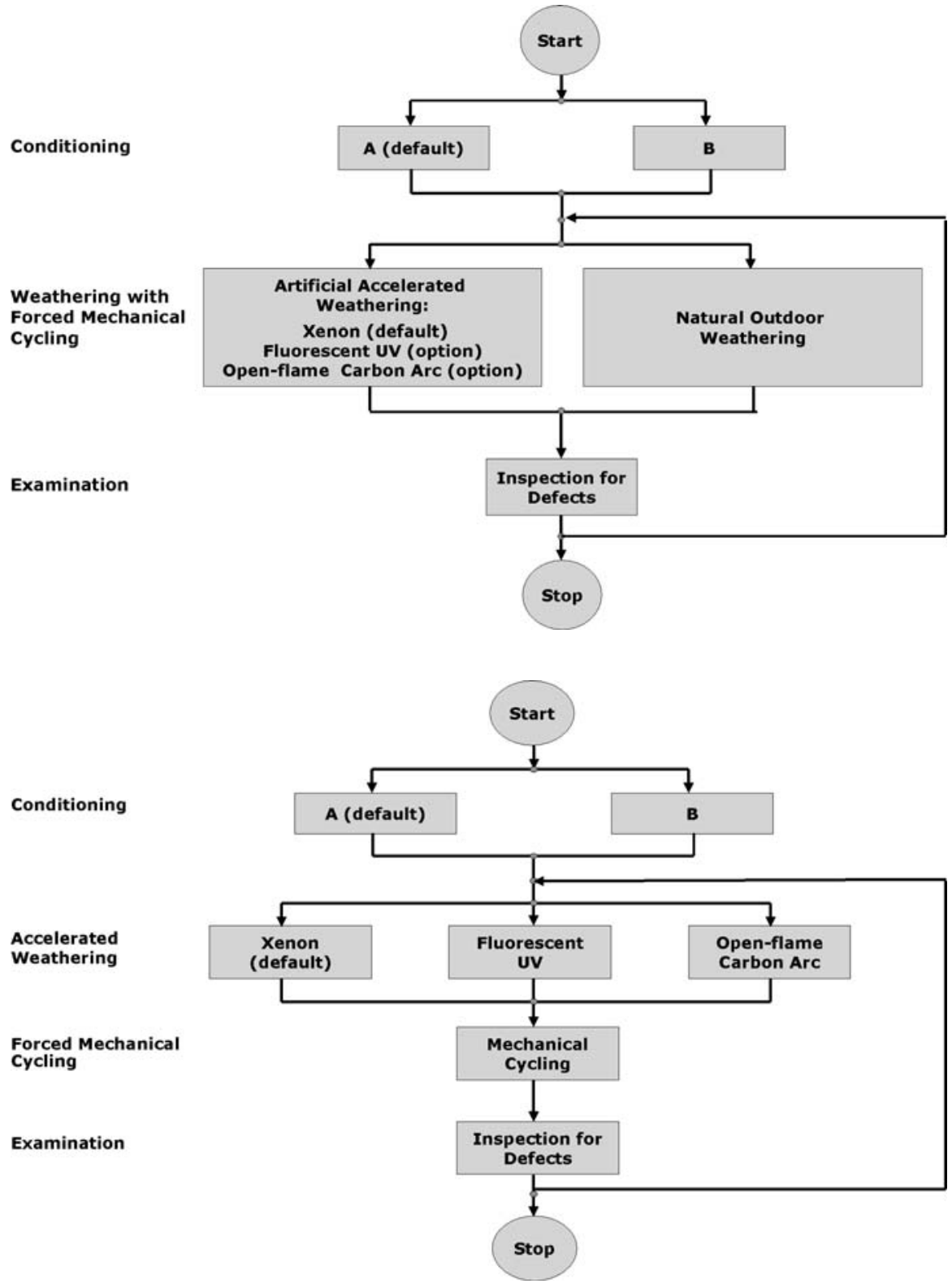

as a predictor of the service life of a sealed joint for a given climate and location and on a given building has not been demonstrated.

\section{Results obtained in preliminary evaluations of proposed durability test methods}

The objective of the work program suggested by the Japanese researchers during the 190-SBJ committee meeting in 2001 was to develop an accelerated artificial weathering test method and to benchmark the results obtained with this method against the results of a long-term outdoor weathering study. The proposed experimental plan comprised accelerated weathering in three different devices based on xenon arc, open flame carbon arc, and fluorescent ultraviolet light sources for up to $10,000 \mathrm{~h}$ duration as well as outdoor weathering at three exposure sites at Southern, Central and Northern locations in Japan over a period of 20 years. The Japanese researchers were convinced that simultaneous weathering and 
mechanical cycling would accelerate the degradation rate in sealants and suggested the novel specimen as a key tool in this approach. The following provides a short summary of the current stage of this study, conducted under the auspices of RILEM TC 190-BJ, as well as the preliminary findings reported by the Japanese experts at RILEM meetings and ASTM symposia $[9,22]$.

One of the first questions to address in the study was the optimum rate at which the extension and compression positions in the specimen should be switched. Therefore, during the 2001-2002 period, a preliminary natural weathering experiment was carried out with two sealants being exposed for 12 months on an outdoor exposure rack located in Yamanashi Prefecture in Japan facing south at an inclination angle of $45^{\circ}$. The sealant specimens were exposed to cyclic movements using three different switching frequencies (once/month, once/week and twice/week), and additionally, as a reference, exposed to no movement at all (fixed position without switching).

Exposing the specimens to movement had a statistically significant effect on the degree of degradation when compared to static exposure (no movement). However, no significant difference was observed between the specimens exposed to movements with different switching frequencies. With this result, the Japanese researchers in 2002 proposed a switching frequency in the outdoor test procedure of once/month, giving consideration to work efficiency. For the accelerated weathering exposure, a switching interval of $500 \mathrm{~h}$ was proposed.

The test protocol discussed in 2002 as the basis of the Japanese study then comprised 24 commercially available sealants of seven chemical types exposed in three accelerated weathering equipments and at three outdoor exposure sites. Tables 1 and 2 summarize the experimental parameters for this study.

The Japanese researchers proposed using the ISO 4628 classification scheme, which was developed for the assessment of the surface cracking of degraded coatings, as a rating system for the degradation observed on the sealant specimens [24]. Tables 3 and 4 provide information on the ISO 4628 classification schemes for quantity and size of cracks.

In their initial evaluations, the Japanese experts then calculated the product of quantity $(\mathrm{Q})$ and size (S) of surface cracks, termed "degree of degradation" (DoD), as the indicator of degradation induced by weathering, as shown in Eq. 1 below.

Table 1 Experimental conditions of Japanese (JAI initiated) exposure tests

\begin{tabular}{|c|c|c|c|c|}
\hline \multirow{2}{*}{$\begin{array}{l}\text { Exposure } \\
\text { parameters }\end{array}$} & \multirow{2}{*}{$\begin{array}{l}\text { Outdoor } \\
\text { exposure }\end{array}$} & \multicolumn{3}{|l|}{ Accelerated exposure } \\
\hline & & $\begin{array}{l}\text { Xenon arc with } \\
\text { daylight filters }\end{array}$ & $\begin{array}{l}\text { Open flame carbon arc with } \\
\text { Type } 3 \text { filter; see Table } 1 \text { in } \\
\text { ISO } 4892-4(2004) \text { [23] }\end{array}$ & $\begin{array}{l}\text { Fluorescent } \\
\text { UVA-340 lamp }\end{array}$ \\
\hline $\begin{array}{l}\text { Duration } \\
\quad \text { (planned) }\end{array}$ & 20 years & & $5,000 \mathrm{~h}$ & \\
\hline $\begin{array}{l}\text { Switching } \\
\text { interval } \\
\text { for mechanical } \\
\text { cycling }\end{array}$ & 1 month & & $500 \mathrm{~h}$ & \\
\hline Conditions & $\begin{array}{l}\text { (Location within } \\
\text { Japan) } \\
\text { - North } \\
\text { - Central } \\
\text { - South } \\
\text { - (Angle): } 45^{\circ}\end{array}$ & $\begin{array}{l}\text { (Irradiation) } \\
\text { - } 550 \mathrm{~W} / \mathrm{m}^{2} \text { at } \\
290-800 \mathrm{~nm} \\
\text { - b.p.: } 63^{\circ} \mathrm{C} \\
\text { - r.h.: } 50 \% \\
\text { - } 102 \mathrm{~min} \text { irradiation } \\
\text { followed by } 18 \text { min } \\
\text { irradiation plus water } \\
\text { spray }\end{array}$ & $\begin{array}{l}\text { (Irradiation) } \\
\text { - } 255 \mathrm{~W} / \mathrm{m}^{2} \text { at } 300-700 \mathrm{~nm} \\
\text { - b.p.: } 63^{\circ} \mathrm{C} \\
\text { - r.h.: } 50 \% \\
\text { - } 102 \text { min irradiation } \\
\text { followed by } 18 \text { min } \\
\text { irradiation plus water } \\
\text { spray }\end{array}$ & $\begin{array}{l}\text { (Irradiation) } \\
\text { - } 25 \mathrm{~W} / \mathrm{m}^{2} \text { at } \\
300-400 \mathrm{~nm} \\
\text { - b.p.: } 63^{\circ} \mathrm{C} \text { (on) } \\
\text { - } 50^{\circ} \mathrm{C} \text { (off) } \\
\text { - } 4 \mathrm{~h} \text { irradiation at a b.p. } \\
\text { of } 63^{\circ} \mathrm{C}, 4 \mathrm{~h} \text { dark } \\
\text { with condensation at } 50^{\circ} \mathrm{C}\end{array}$ \\
\hline
\end{tabular}

b.p.: (uninsulated) black panel thermometer, r.h.: relative humidity, (on), (off): lamps switched on and off 
Table 2 Geographic and climatic features of outdoor test locations in Japan

\begin{tabular}{|c|c|c|c|}
\hline Exposure parameters & North & Central & South \\
\hline Latitude & $43^{\circ} 46^{\prime} \mathrm{N}$ & $35^{\circ} 43^{\prime} \mathrm{N}$ & $24^{\circ} 44^{\prime} \mathrm{N}$ \\
\hline $\begin{array}{l}\text { Average maximum } \\
\text { temperature }\left({ }^{\circ} \mathrm{C}\right)\end{array}$ & 11.8 & 18.8 & 26.2 \\
\hline $\begin{array}{l}\text { Average minimum } \\
\text { temperature }\left({ }^{\circ} \mathrm{C}\right)\end{array}$ & 2.2 & 10.6 & 21.0 \\
\hline Precipitation (mm/year) & 1,150 & 1,481 & 2,058 \\
\hline $\begin{array}{l}\text { Amount of UV radiation }{ }^{\mathrm{a}} \\
\left(0^{\circ}, \mathrm{MJ} / \mathrm{m}^{2} \text { in year }\right)\end{array}$ & 240 & 313 & 367 \\
\hline
\end{tabular}

${ }^{\text {a }}$ Wavelength: 315-400 $\mathrm{nm}$

Table 3 Rating scheme for designating the quantity of cracks

\begin{tabular}{ll}
\hline Rating & Quantity of cracks, Q \\
\hline 0 & None, i.e. no detectable cracks \\
1 & Very few, i.e. some just significant cracks \\
2 & Few, i.e. small but significant amount of cracks \\
3 & Moderate, i.e. medium amount of cracks \\
4 & Considerable, i.e. serious amount of cracks \\
5 & Dense, i.e. dense pattern of cracks \\
\hline
\end{tabular}

Table 4 Rating scheme for designating the size of cracks

\begin{tabular}{ll}
\hline Rating & Size of cracks, $S$ \\
\hline 0 & Not visible under $\times 10$ magnification \\
1 & Only visible under magnification up to $\times 10$ \\
2 & Just visible with normal corrected vision \\
3 & Clearly visible with normal corrected vision \\
4 & Large cracks generally up to $1 \mathrm{~mm}$ wide \\
5 & Very large cracks generally more than $1 \mathrm{~mm}$ wide \\
\hline
\end{tabular}

Degree of degradation $(\mathrm{DoD})$

$=$ Quantity $(\mathrm{Q}) \times$ Size $(\mathrm{S})$

Preliminary evaluations based on 2- and 3-year outdoor exposure and 3,000 and 5,000 h of laboratory-accelerated exposures indicate that the effect of movement varies with the type of sealant. As discussed previously, weathering with cyclic tensile compression/extension movements could not be compared with weathering under static conditions based on the Japanese study. Rather the sections of the test specimen undergoing cyclic movement were compared to the central portion of the sealant, which was subjected to some shear movements.
Furthermore, the degradation evaluated in terms of the degree of degradation (DoD) may not be a good measurement of the actual degradation. However, for most of the sealants, the data showed that cyclic tensile compression/extension movements increased the degree of degradation by only twofold or less and for some sealants it had no effect on the degree of degradation. Furthermore, within the preliminary results there was very little difference between the $15 \%$ and $30 \%$ cyclic tensile movements amplitude based on the DoD measure. These findings were similar for the outdoor and laboratory accelerated exposures.

\section{Intentional deviations between the ongoing Japanese study and the test protocols recommended by TC190-SBJ}

In October 2006, 19 experts within ISO TC59/SC8 Work Group 10 'Aesthetic Issues' evaluated twelve samples of degraded sealants, taken from actual service joints, based on the ISO 4628 classification scheme, however, without the use of pictorial references. Based on the ratings given for size and quantity of cracks, the degree of degradation was calculated as described above. The round-robin test showed a strong dependency of the ratings, especially for the combined measure of degree of degradation, on the perceptual and psychological biases of the observer [25].

Ongoing discussions within this work group suggest that the development of sealant-specific pictorial references may reduce, but certainly not eliminate, the inherent limitations associated with the visual inspection of specimens. Efforts made in the recent past at overcoming these human limitations by using state-of-the-art optical imaging and software analysis technologies have been at least partially successful. For outdoor weathered sealants, it has been shown that the density of cracks and the average crack width, when considered together, appropriately describe cracking severity in a quantitative manner and that surface cracking generally can be well characterized using an automated digital image capturing and processing system [26]. The Japanese researchers within RILEM TC190-SBJ have also initiated work on automated evaluation systems with the objective of deriving a quantitative assessment of 
the cracking and crazing type of surface degradation in sealants.

The compromise reached within TC190-SBJ is to include a visual assessment of the quantity and size of cracks in the recommended test methods; however, not to calculate the derived degree of degradation value, since the multiplication of the two ratings only compounds the error in the assessment. Furthermore, the users of the RTR are expected to document the degraded surface of the sealant specimens using highresolution digital photography.

The RTR methods deviate in further details from the ongoing Japanese study. In the Japanese study, test specimens are exposed to an accelerated weathering cycle in the fluorescent ultraviolet light based automated weathering equipment consisting of $4 \mathrm{~h}$ of ultraviolet light at $63^{\circ} \mathrm{C}$ and $4 \mathrm{~h}$ of dark (light being switched off) at $50^{\circ} \mathrm{C}$. The dark cycle at the lower temperature is expected to induce condensation of moisture on the test specimens. However, as mentioned earlier, it has been shown by researchers within the ASTM C24 committee 'Seals and Sealants' that the time-of-wetness is very limited in this weathering cycle for samples with a large mass and/or high heat capacity. The RTR test method for accelerated weathering using the fluorescent ultraviolet light equipment therefore requires water spray instead of relying on condensation to occur. Furthermore, the RTR specifies a cycle based on $8 \mathrm{~h}$ dry and $4 \mathrm{~h}$ wet (spray) exposures, with the ultraviolet light being switched on continuously during both dry and wet periods. Thus, in the Japanese study, the specimens exposed in the fluorescent light equipment experience less ultraviolet light and more water than in the RTR method. However, the reasoning for the cycle specified in the RTR method for the fluorescent light exposure equipment $(66.7 \%$ dry, $33.3 \%$ wet) was to make it more comparable to the exposures experienced in the xenon arc and the open flame carbon arc devices (85\% dry, $15 \%$ wet).

The fluorescent light accelerated weathering device in the Japanese study was set to operate at an irradiance of $25 \mathrm{~W} / \mathrm{m}^{2}$ for the $300-400 \mathrm{~nm}$ spectral region, while the RTR method specifies an irradiance setting of $0.75 \mathrm{~W} /\left(\mathrm{m}^{2} \mathrm{~nm}\right)$ at $340 \mathrm{~nm}$, which corresponds to $41 \mathrm{~W} / \mathrm{m}^{2}$ for the $300-400 \mathrm{~nm}$ region.

The RTR method requires the open-flame carbonarc light source to be fitted with daylight filters in order to reduce the short-wave UV irradiance in an attempt to obtain a better simulation of the short wavelength UV region of daylight. The Japanese study, however, used extended UV filters that transmit more short wavelength UV light and which sometimes are used to accelerate test results. Although this type of filter has been specified in many tests because of historical precedent, the extended UV filters transmit substantial radiant energy below $300 \mathrm{~nm}$, which is the typical cut-on wavelength for terrestrial sunlight. Therefore, use of extended UV filters may result in ageing processes not occurring outdoors.

Further deviations can be found in the specification of the thermometers and the resulting actual exposure temperatures. The Japanese study specifies use of the uninsulated black panel thermometer and an operating temperature set point of $63^{\circ} \mathrm{C}$. Under given operation conditions, the uninsulated black panel thermometer tends to indicate lower temperatures than the insulated black standard thermometers. The temperature difference between the two ranges between 3 and $12^{\circ} \mathrm{C}$, being smaller at lower irradiance levels. For the RTR method, the black standard (insulated black panel) thermometer was specified as the default thermometer and the temperature set point as $65^{\circ} \mathrm{C}$. The black standard thermometer as default was chosen as a default because of consistency with other ISO and ASTM standards. The higher operating temperature set point was chosen since it better represents the temperature exposure in actual curtainwall service joints. Since fluorescent UV lamps emit very little infrared radiation when compared to xenon arc or carbon arc sources, the primary heating of the specimen surface in the fluorescent UV light apparatus is by convection from heated air passing across the specimen. Therefore, for the fluorescent UV light apparatus, there is a minimal difference between the temperatures of an insulated or uninsulated black thermometer, specimen surface, air in the test chamber, and different coloured specimens.

Another key difference between the Japanese study and the accelerated weathering RTR is the frequency at which the extension/compression position in the specimen is switched. The Japanese researchers chose a switching interval of $500 \mathrm{~h}$ in their accelerated weathering protocols, with the full extension and compression cycle then taking 1,000 $\mathrm{h}$ [9]. Based on the initial results reported by the 
Japanese researchers, it was felt that the degradation induced by cyclic movement versus that induced by weathering factors in the Japanese accelerated ageing protocol did not sufficiently balance these factors to mimic the degradation occurring in actual weatherproofing service joints. Already previously, during the development of the RILEM TC139-DBS accelerated weathering durability test method [2], it was noticed that the cyclic movement degradation had to be increased in the accelerated ageing protocol by including a certain amount of fatigue cycling [3, 4]. Therefore, the committee decided to shorten the movement cycle in the TC190-SBJ accelerated weathering method to $96 \mathrm{~h}$ (4 days). Thus, the RTR now proposes the following weekly mechanical exposure cycle: 'Mechanical cycling is achieved by extending one end of the test specimen at a rate of $5.5 \pm 0.7 \mathrm{~mm} / \mathrm{min}$, then holding this extension for $48 \mathrm{~h}$. After this period, the test specimen is allowed to relax for $5 \mathrm{~min}$ before the other end of the test specimen is extended at a rate of $5.5 \pm 0.7 \mathrm{~mm} / \mathrm{min}$. This extension is again held for $48 \mathrm{~h}$. Then the test specimens are kept in a relaxed (unstressed) state for $72 \mathrm{~h}$ (3 days). The above described weekly mechanical exposure cycle is repeated for a total of 6 weeks'. This movement cycle represents roughly a 10 -fold increase in movement exposure versus the Japanese study. However, further work will be required during the evaluation phase of the proposed RTR test method to determine whether this amount of mechanical cycling is sufficient to achieve a high acceleration of degradation while maintaining good correlation with the type of degradation (failure mode) occurring in actual service joints. For the natural outdoor weathering protocol, the duration of one mechanical movement cycle was maintained at 2 months, as is the case in the Japanese study.

\section{Conclusions}

The new RILEM Test Recommendations (RTR) provide test protocols for accelerated artificial and natural outdoor weathering that allow simultaneous exposure to weathering and movement cycles. Furthermore, the RTR methods allow an estimation of the long-term movement capability of sealants while taking the effects of weathering and long-term mechanical cycling directly into account.
The ongoing Japanese study indicates that the proposed RTR methods are able to differentiate between products with regard to their resistance to accelerated and outdoor ageing and mechanical cycling. The type of failure and the changes in surface appearance observed during the test regimes are similar to those observed in actual service conditions.

The efficiency of the new test methods based on the newly developed flex-joint test specimen has been confirmed. This new test specimen allows a reduction in the total number of specimens employed by exposing the sealants simultaneously in a single specimen to compression and extension movements. It also simplifies the handling operations needed to induce the cyclic movement.

However, the committee strongly encourages further studies in order to evaluate the durability test methods proposed in the RILEM Technical Recommendations. Substantial further work will be required to establish acceleration factors for various sealant products and correlation with the performance of these products in actual service joints. In addition, replacement of static cure with dynamic cure that simulates real time movement parameters as well as inclusion of backer rod in the specimen design should be investigated in order to provide more realistic evaluation of performance.

\section{References}

1. Wolf AT (1999) Progress toward the development of a durability test method for sealants. In: Wolf AT (ed) Durability of building sealants, RILEM report 21. RILEM Publications, Bagneux, pp 365-380

2. Wolf AT (2001) Durability test method-determination of changes in adhesion, cohesion and appearance of elastic weatherproofing sealants for high movement façade joints after exposure to artificial weathering. Mater Struct 34:579-588

3. Jones TGB, Hutchinson AR, Wolf AT (2001) Experimental results obtained with proposed RILEM durability test method for curtain wall sealants. Mater Struct 34:332-341

4. Miyauchi H, Enomoto N, Sugiyama S, Tanaka K (2004) Artificial weathering and cyclic movement test results based on the RILEM TC139-DBS durability test method for construction sealants. In: Wolf AT (ed) Durability of building and construction sealants and adhesives. STP 1453. ASTM International, West Conshohocken, pp 206-212

5. Bolte H, Boettger T, Wolf AT (1999) Preliminary report on the ageing behaviour of sealants exposed to dynamic natural and artificial weathering. In: Wolf AT (ed) Durability 
of building and construction sealants. RILEM Publications, Bagneux, pp 151-172

6. Anonymous (2002) ISO 11600-building constructionsealants-classification and requirements. International Standardisation Organisation, Geneva

7. Anonymous (2005) ASTM C920-05 standard specification for elastomeric joint sealants. ASTM International, West Conshohocken

8. Anonymous (2001) RILEM TC190-SBJ document N005proposal of new weathering test method (by Japanese experts). Presentation by Noriyoshi Eonomoto (2001-09-10)

9. Enomoto N, Ito A, Shimizu I, Matsumura T, Takane Y, Tanaka K (2007) Study of weatherability of construction sealants with novel testing method. In: Wolf AT (ed) Durability of building and construction sealants and adhesives, 2nd edn. STP1488. ASTM International, West Conshohocken, pp 82-90

10. Anonymous (2007) ISO TC59/SC8/WG06 document N012-durability test method-minutes of meeting held at DIN, Berlin, on 09 October 2006. International Standardisation Organisation, Geneva

11. Anonymous (2004) ASTM C1519-04 standard practice for evaluating durability of building construction sealants by laboratory accelerated weathering procedures. ASTM International, West Conshohocken

12. Iglauer S, Hutchinson AR, Lee TCP (2004) Effect of foam backer rod on the deep section cure of one-part sealants and Effect of foam backer rod on the mechanical performance of sealed joints. In: Wolf AT (ed) Durability of building and construction sealants and adhesives. STP1453. ASTM International, West Conshohocken, pp 171-183, 184-205

13. Anonymous (1989) Recommendations for the integrated irradiance and the spectral distribution of simulated radiation for testing purposes; solar spectral irradiance. CIE Publication No. 85, Commision Internationale de L'Eclairage (CIE), Central Bureau, Vienna, Austria, ISBN 3900-734-224

14. Brennan P, Everett ET (1999) Light sources used in laboratory weathering of sealants and their effect on the correlation with natural weathering. In: Wolf AT (ed) Durability of building sealants, RILEM report 21. RILEM Publications, Bagneux, pp 155-172

15. Wypych G, Kuberski S, Lee F (2003) Sealant failure morphology - the important criterion of equipment selection for weathering studies. In: Wolf AT (ed) Science and technology of building seals, sealants, glazing and waterproofing. ASTM STP 1453. ASTM International, West Conshohocken, pp 310-321

16. Searle ND (2003) Harmonization and optimization of weathering test methods for building and construction sealants and adhesives. In: Wolf AT (ed) Science and technology of building seals, sealants, glazing and waterproofing. ASTM STP 1453. ASTM International, West Conshohocken, pp 355-371

17. Anonymous (2002) ISO 11431-building constructionjointing products-determination of adhesion/cohesion properties of sealants after exposure to heat, water and artificial light through glass. International Standardisation Organisation, Geneva

18. Crewdson MJ (1999) Exposure and mounting techniques used in the outdoor weathering of sealants. In: Wolf AT (ed) Durability of building sealants, RILEM report 21 . RILEM Publications, Bagneux, pp 173-180

19. Haacke G, Longordo E, Brinen JS, Andrawes FF, Campbell BH (1999) Chemisorption and physical adsorption of light stabilisers on pigment and ultrafine particles in coatings. J Coat Technol 71:87-94

20. Vandereecken P, Wolf AT, Bolte H, Boettger T (1999) FTIR spectroscopic investigation of the out-door degradation of polysulfide sealants. In: Wolf AT (ed) Durability of building and construction sealants. RILEM Publications, Bagneux, pp 243-258

21. Lee TCP, Wolf AT (1999) Factors governing the durability of sealant formulations. In: Wolf AT (ed) Durability of building sealants, RILEM report 21. RILEM Publications, Bagneux, pp 203-223

22. Enomoto N (2006) Test results for weatherability of sealants. Presentation at RILEM TC190-SBJ meeting, Berlin, Germany, 9 October 2006

23. Anonymous (2004/2005) ISO 4892-4:2004/Cor 1:2005plastics - methods of exposure to laboratory light sources-part 4: open-flame carbon-arc lamps. International Standardisation Organisation, Geneva

24. Anonymous (2003) ISO 4628-4 paints and varnishesevaluation of degradation of coatings-designation of quantity and size of defects, and of intensity of uniform changes in appearance-part 4: assessment of degree of cracking. International Standardization Organization, Geneva

25. Wolf AT (2007) ISO/TC 59/SC 8/WG 10 document N 010 - detailed findings of round robin visual evaluation of surface degraded sealant samples (during meeting at DIN, Berlin on 2006-10-10). International Standardisation Organisation, Geneva

26. Wolf AT, Sugiyama S, Lee F (2007) Use of optical imaging/image analysis system for the quantitative analysis of surface changes induced by outdoor weathering on sealants. In: Wolf AT (ed) Durability of building and construction sealants and adhesives. STP1488. ASTM International, West Conshohocken, pp 102-112 